\title{
COMMUNICATING THROUGH MUSIC: CREATING ACCESSIBLE SONGS FOR INDIVIDUALS WITH MEMORY LOSS Whint
}




\title{
COMMUNICATING THROUGH MUSIC: CREATING ACCESSIBLE SONGS FOR INDIVIDUALS WITH MEMORY LOSS
}

\author{
by \\ Hallie Anthony \\ Bachelor, Media Production, Ryerson University, 2015
}

\author{
A MRP \\ presented to Ryerson University \\ in partial fulfillment of the \\ requirements for the degree of \\ Master of Arts \\ In the Program of \\ Master of Professional Communication
}

Toronto, Ontario, Canada, 2017

CHallie Anthony, 2017 


\section{i. Author's Declaration for Electronic Submission of a MRP}

I hereby declare that I am the sole author of this MRP. This is a true copy of the MRP, including any required final revisions.

I authorize Ryerson University to lend this MRP to other institutions or individuals for the purpose of scholarly research.

I further authorize Ryerson University to reproduce this MRP by photocopying or by other means, in total or in part, at the request of other institutions or individuals for the purpose of scholarly research.

I understand that my MRP may be made electronically available to the public. 


\section{COMMUNICATING THROUGH MUSIC: CREATING ACCESSIBLE \\ SONGS FOR INDIVIDUALS WITH MEMORY LOSS}

by

Hallie Anthony

Bachelor, Media Production, Ryerson University, 2015

\section{ii. ABSTRACT}

This Major Research Paper (MRP) is a research-creation project that explores the role, impact and influence of music on human communication. More specifically, this MRP investigates the way that music can assist in cognitive comprehension and information retention in individuals with varied forms of memory loss and considers the ways that music can be used to assist in aiding verbal memory and communicating emotion. It also offers insight on methods that could be utilized to further study this area of research. As a research-creation project, this MRP includes three original musical compositions ( $\underline{\text { Composition } \mathrm{A}^{1}}$, $\underline{\text { Composition } \mathrm{B}^{2}}$, and $\underline{\text { Composition }}$ $\left.\underline{C}^{3}\right)$ that were developed in response to the scholarly literature on this topic. The three musical compositions that have been produced for this project aim to communicate emotion, create aesthetic satisfaction, and aid in

\footnotetext{
${ }^{1}$ https://youtu.be/N8uVng3vcXg

${ }^{2}$ https://youtu.be/TO8diVtQGuY

${ }^{3}$ https://youtu.be/2k1SzofzC9U
} 
reinforcing the production of memories based on the information presented in the compositions. The composition creation process was informed by information gathered throughout the research process that addresses topics such as: the influence of music on individual listeners, emotion in music, and musical techniques that can be used to successfully communicate ideas. Beyond the scope of this MRP/research-creation project, these compositions aim to serve as a basis for testing cognitive function in individuals with varied forms of memory loss. Detailed discussion of the design of the musical compositions, including information related to the composition influences, and a breakdown of each composition are included alongside the MRP's focus on scholarly literature and research. Furthermore, the potential implications of these compositions, including the ways they could assist in future research, is discussed. 


\section{iii. ACKNOWLEDGEMENTS}

I would like to thank my supervisor, Dr. Matthew Tiessen, for his constant reassurance and guidance throughout this process, and my second reader, Dr. Marty Fink, for offering valuable feedback. 


\section{TABLE OF CONTENTS}

i. Author's Declaration .................................................... 3

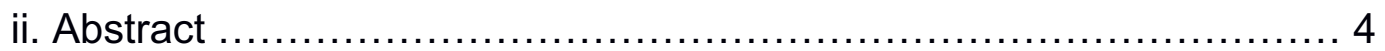

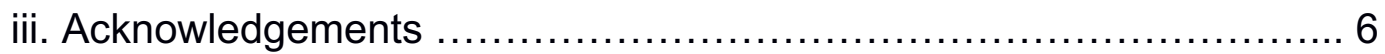

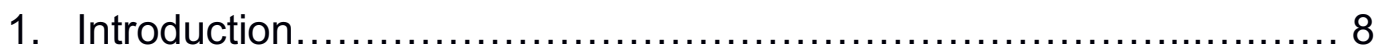

1.1. About the Author: A disclaimer ......................... 9

2. Research Questions ................................................11

3. Literature Review ...................................................

3.1. Music as a Source of Emotion and Meaning …......... 13

3.2. Music as a Communicative Tool ......................... 17

3.3. Music, Memory, and Information Retention ............. 21

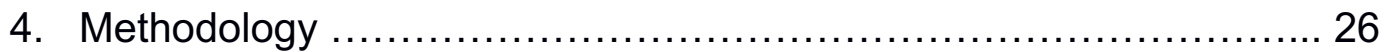

5. The Compositions: An Overview ................................... 28

5.1. Composition Influence from the Literature ............... 29

5.2. Composition Breakdown ................................ 34

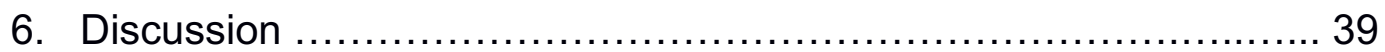

6.1. General Notes on Creating the Compositions ........... 39

6.2. Individual Composition Summary...................... 42

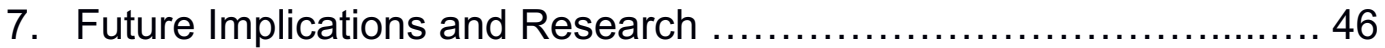

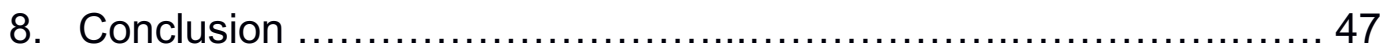




\section{Introduction}

Exploring the ways that music communicates and the way it can assist with cognitive comprehension and information retention is the focus of this Major Research Paper (MRP) and research-creation project. More specifically, this project responds to the question: How can music be used as a communicative tool that assists in aiding verbal memory and communicating emotion? The objective here is to develop critical and creative insights on techniques that could assist in the development of music capable of effectively communicating to those with memory deficiencies, specifically those with dementia or Alzheimer's disease, and to aid them in memory rehabilitation.

For this project, three musical compositions between 50 and 100 seconds have been produced, the aim being to communicate emotion, create aesthetic satisfaction, and aid in verbal memory of the information presented in the compositions. The creation of the compositions has been informed by information gathered throughout the research process that speaks to issues such as: the influence of music, emotion in music, musical factors that work to communicate ideas, and more. Beyond the scope of this MRP, these compositions aim to serve as a basis for testing cognitive function and memory in individuals with varied forms of memory loss.

This MRP includes three primary sections. The first section is a literature review that explores music and the impact it has on emotion, 
music's relationship to advertising and consumer behaviour, musical elements that contribute to music's communicative ability, and finally, the relationship between music, memory, and information retention. Furthermore, this first section contributes to an understanding of the communicative power of music and to the relationship music has with listeners. The second section is comprised of a detailed discussion of the design of the accompanying musical compositions, including information related to the composition influences, and a breakdown of each composition. The third section discusses the future implications of these compositions, including the ways they could assist in future research, and how they could be tested beyond the scope of this MRP.

\subsection{About the Author: A Disclaimer}

Before going further, I feel it is critical to make readers aware of my musical background, since my personal experiences related to music have informed the creation of these compositions. I was born into a musical family and from a young age was heavily involved with music. My grandfather was a pianist, my mother plays clarinet and piano, my brother plays tenor saxophone, and over the years I have been strongly drawn to string instruments. At age $14 \mathrm{I}$ received my first guitar and began composing original compositions shortly thereafter. In the subsequent years, I picked up piano, ukulele, and beginner knowledge of banjo and mandolin. I continue to write and compose to this day and found fortune a few years ago when a composition I created made it to radio. Though I love all genres 
of music, I am most familiar with rock, pop, and folk genres that typical follow four-chord progressions, often with an accompanying and often uncomplicated melody. I am self-taught, with intermediate knowledge of musical theory.

Personal curiosity of the role music plays in communicating emotion and the ways it could assist in communicating messages prompted my interest in this research area. In addition, one of my family members was recently diagnosed with vascular dementia, a type of dementia that causes memory loss, resulting in difficulty speaking and remembering everyday tasks, such as teeth brushing. After hearing of the issues he was facing, I was inspired to explore music as a communicative medium, particularly the influence music could have on memory and information retention. As a result, I decided to create original compositions that aimed to communicate ideas about everyday activities, in hopes that they could one day be used to assist in memory recall. The lyrical theme of these compositions was inspired by my family member's difficulty with everyday tasks (e.g., brushing his teeth, shaving, etc.) and a detail mentioned in Simmons-Stern, Budson, and Ally's (2010) article describing the daughter of an Alzheimer's patient using music to teach her father about current news stories. This piqued my interest and led me to further explore this area of research.

The three compositions developed for this MRP: (1) have been composed and recorded solely by myself, (2) involve instruments that I have experience playing, (3) are expressed and explained through beginner to 
intermediate language of musical notation and theory, (4) involve my interpretation of content found in the research, which has informed the creation of the compositions, (5) fall within the pop, rock, and/or folk genres popular in North America, and (6) are rooted in Western music culture. As a result, these compositions are necessarily informed by my own experiences of musical genres, my own familiarity with certain types of instrumentation, and my personal theoretical and practical knowledge of music. At the same time, the compositions are also creative distillations of complex ideas. My broad objective, then, has been to draw on scholarly research to create effective communication tools capable of reaching listeners whose ability to remember everyday routines has been impaired and who, perhaps by having catchy melodies stuck in their head, might become empowered to rediscover their abilities to perform everyday tasks.

\section{Research Questions}

Examining the ways that different musical styles and techniques can influence and affect individuals could assist with understanding the potential impact that music has on effectively communicating to listeners. This research project addresses and explores the ways that music can influence cognitive comprehension and information retention, aiding verbal memory and communicating emotion, and was guided by the following questions: 
1. Is music a valuable tool to use when attempting to communicate complex ideas, such as emotion or specific tasks?

2. Which musical traits and techniques (tempo, timbre, musical mode, repetition, etc.) contribute to cognitive comprehension, information retention and the aiding of verbal memory in individuals with varied forms of memory loss?

Responding to these questions has assisted in my understanding of the impact that music has on communicating complex ideas and has allowed me to develop a deeper understanding of the most effective methods to use when communicating with individuals through music. The three compositions I have developed during this research-creation project are the products of this exploration of musical affordances.

\section{Literature Review}

In the early twentieth century, music psychologists proposed a complex way of understanding musical pitch, resulting in a stronger focus on music education and sparking discussions and studies by theorists across the globe (Révész, 1954; Welleck and Baker, 1926). This section offers a review of the literature that has been developed since then, focusing specifically on three main areas of research: (1) music as a source of emotion and meaning, (2) music as a communicative tool, and (3) the relationship between music, memory, and information retention. 


\subsection{Music as a Source of Emotion and Meaning}

This section of the literature review explores music as a source of emotion and meaning. More specifically, it discusses and reviews scholarly works that speak to the ways human emotion is influenced and affected by music, and the ways that emotional meaning is created through musical engagement.

Music is a communicative tool that can be used to evoke emotions in listeners (Koelsch, 2010; Panksepp and Bernatzky, 2002). Panksepp and Bernatzky (2002) discuss the influence music has on the brain with an emphasis on the effect music has on emotion. They focus on the ways musical information can influence and speak to various parts of the brain and the emotional systems present within it (Panksepp and Bernatzky, 2002). Panksepp and Bernatzky (2002) explain that the degree to which music influences emotion differs in individuals and is likely a result of a combination of many complex factors that are still not fully understood. In their discussion of the relationship between music and emotion, they draw attention to a gap in research on music as an affective medium, suggesting that investigating this area of research could eventually teach us "how music might be used more effectively in our educational and therapeutic initiatives" (Panksepp and Bernatzky, 2002, p. 152).

Illie and Thompson (2006) discuss music as a source of emotion and meaning, along with the concept of 'prosody,' which "refers to the use of intonation (pitch variation) and rhythm (stress and timing) in speech, as 
distinct from verbal content" (p. 319). They explain that the pairing of music and prosody is what works to communicate emotional meaning (Illie and Thompson, 2006). Similarily, Juslin and Laukka (2001) examine the impact vocal intonation and acoustic cues have on the emotion a listener perceives a musical composition to be communicating. They suggest that vocal expression, the emotion and intensity present in one's speech, influences the perceived emotion of what is being stated (Juslin and Laukka, 2001) and offer evidence suggesting the importance of vocal expression when attempting to communicate a specific emotion.

Although, as mentioned earlier, this paper primarily focuses on Western musical traditions, there is evidence of universal musical traits related to emotion and meaning. Accordingly, Fritz et al. (2009) investigate whether similar emotions are universally experienced when encountering certain types of musical compositions, considering the presence of emotion in music on a cross-cultural level. The authors explore whether the importance of emotional expression in music is universal among other cultures, using a native African population and a Western population as points of comparison (Fritz et al., 2009). The results of Fritz et al.'s (2009) study reveal that, in addition to the Western population, the non-Western population experiences the musically-induced emotions of happy, sad, and scared/fearful when listening to different types of music. Their discovery is an important factor to consider when creating compositions. Since these are the emotions evidenced to be universal, these are the three emotions 
my compositions aim to communicate. If future research in this area allows for the testing of these compositions, it will be important to consider the universal perception of emotions by other cultures.

\subsubsection{Music, advertising, emotion, and behaviour.}

Existing literature and research supports the notion that music is a source of emotion and meaning, and emphasizes the importance of music as a communicative tool. Similar views of the power of music arise in studies that focus on music, advertising, emotion, and behaviour.

Studies within the field of advertising contribute to a broader understanding of the impact music used in advertisements has on the emotions and cognitive functions of individuals, serving as a basis - and point of comparison - for understanding the influence it has on individuals outside of the realm of advertising. In advertising, music has been shown to be "an affective background component that causes attachment to the product without the cognitive involvement of the viewer" (Scott, 1990, p. 224). Gorn (1982) focuses on understanding music's influence on behaviour in the realm of advertising by discussing the impact that music used in advertising has on consumer behaviour. His study demonstrates that subjects are more likely to choose an advertised product when it is paired with aesthetically pleasing music, cementing the notion that music does indeed have an impact on consumer behaviour (Gorn, 1982). Awareness of the role that aesthetic appeal plays in music, and the influence it has on the perception of the product or message paired with 
said musical piece, is important to consider when creating a composition composed to communicate a specific message. Gorn's (1982) work was met with critiques later that decade when research by Kellaris and Cox (1989) replicated Gorn's (1982) experiment, but yielded opposing results. The divide between the results of these two studies suggests that there must be other factors related to music's ability to influence listeners, that extend beyond the realm of strictly stylistic musical preference and the aesthetic appeal of a musical piece.

Alpert and Alpert (1990) build off previous research in this area (Gorn, 1982) by seeking to uncover the relationship between music, emotion and behaviour. Their experiments investigate the link between music and consumer behaviour, where various products are presented to subjects in their study accompanied by different styles of music (Alpert and Alpert, 1990). Similar to Gorn's (1982) initial study, the results of Alpert and Alpert's (1990) experiment suggest that music can influence individual behaviour, supporting the role of music as a communicative medium.

Other discussions surrounding music, emotion and behaviour consider emotional responses to music and the influence music can have on behaviour. Morris and Boone's (1998) study investigates music's influence on both, purchase intent and emotional response. Their experiment yields interesting results. In contrast to the study by Alpert and Alpert (1990) which suggests music has an influence on purchasing intent, Morris and Boone's (1998) study suggests otherwise. They note that there 
are "no differences in brand attitude and purchase intent, however on emotional response six out of the 12 advertisements showed significant differences between the music and no-music groups." (Morris and Boone, 1998, p. 518). Again, opposing research results in this field draw attention to a gap in an ability to understand what makes music so persuasive and impactful.

Ilie and Thompson (2006) critique the separation-based research practices used by researchers like Gorn (1982) and Kellaris and Cox (1989), that regard music as an independent affective entity that works in specific ways regardless of the context in which it is studied. Ilie and Thompson (2006) suggest that "the methodology of separating [music] syntactically [has led] to procedures that negate the complex functionality of the music" (p. 225), noting that studies such as those performed by Gorn (1982) fail to consider the importance of the relationship between other factors (visuals, social context, etc.). To fully understand music's role as a communicative medium that influences emotion and facilitates meaning, it is important to consider the multiple factors at play in any musical composition.

\subsection{Music as a Communicative Tool}

This section explores various facets of music's communicative ability by examining the relationship between music and classical conditioning theory, and the importance of aesthetic characteristics and musical elements in compositions. 


\subsubsection{Classical conditioning theory.}

Bierley, McSweeney, and Vannieuwkerk (1985) focus on classical conditioning, a theory that was first put forth by Pavlov in 1927. They explain that classical conditioning is a process that "occurs when an arbitrary stimulus (the conditioned stimulus, or CS) predicts some other stimulus (the unconditioned stimulus, or US). After several trials, a response (the conditioned response, or CR) to the CS develops" (Bierley, McSweeney, and Vannieuwkerk, 1985, p. 316). In other words, classical conditioning is a theory that concentrates on associative relationships that form between stimuli. Much of the discussion concerning the relationship between classical conditioning and music relates to advertising. Apaolaza-lbáñez, Zander and Hartmann (2010) discuss this notion, explaining that in advertising "classical conditioning implies that pairing a product (neutral stimulus) with a well-liked piece of music (unconditioned stimulus) will produce an association between the two, and therefore a preference for the product (a conditioned response)" (p. 3807). The conditioned relationship that can be created between a brand or product and a musical piece is suggestive of the power that music has on creating deep-rooted connections with a listener. In the mind of the listener, an association is made between the melody and tone of a song, and the ideas those musical elements represent. As a result, music is an extremely viable tool to use when attempting to communicate ideas to individuals with varied forms of memory loss. 


\subsubsection{Musical elements and aesthetic appeal.}

Another facet of music's communicative ability is the aesthetic appeal that is present within a musical piece. The aesthetic appeal of a musical composition is often what causes listeners to like, or dislike, a song. Ockelford $(2006 ; 2009 ; 2013)$ speaks about the mechanisms at play that cause a listener to feel a certain way when listening to a piece of music. When there is repetition present in a musical piece, for example, there is a constant sense of expectation being met, leading to a feeling of aesthetic satisfaction and resolution (Ockelford 2006; 2009; 2013). Conversely, if a listener does not like a song, or is left feeling unsatisfied after hearing it, it may cloud their judgment of the message being portrayed in the song. As evidenced through other research (Scherer, 2004), music influences and guides our emotions. When listeners enjoy a song that is included in a media piece there is the potential for enhancement of the communicative ability of the song, so it is important to understand the musical factors at play in a musical composition.

In addition to repetition (Ockelford 2006; 2009; 2013), rhythm is another factor at work in a musical piece that a listener may find aesthetically appealing, contributing to music's ability to connect with individuals. Repp (2005) speaks of sensorimotor synchronization which is related to the human ability to hear and feel rhythm, noting that "temporal expectations" are created as a result of "internal synchronization" when music is heard (p. 969). Repp's (2005) article offers complex insight, 
considering the relationship between cognitive function, motor skills, and sensorimotor synchronization. When discussing previous studies, Repp (2005) notes that there is evidence of a relationship between tapping and auditory sensory memory. Findings such as these allude to the importance of rhythm in a musical piece, and the role it can play in assisting with memory and enhancing cognitive function.

In line with Repp (2005), Patel and Daniele (2003) discuss the evolution of musicality in humans with specific emphasis on an exploration of rhythm, examining the relationship between musical rhythm and linguistic rhythm. In a later work, Patel (2006) questions the capability of animals to produce rhythm, further exploring the subject of innate beat perception and synchronization in individuals. Works such as those by Patel (2006), Patel and Daniele (2003) and Repp (2005) offer insight on music's communicative capacity and help us understand the musical factors that contribute to it. If all humans have an inherent sense of rhythm, for example, this could be why we find musical pieces with steady rhythms so aesthetically pleasing. Being aware of the aesthetic satisfaction that can result from a steady rhythm is crucial to understand, as rhythm is a critical element of any musical composition.

Other literature supports the importance of rhythm in musical experience, suggesting that rhythm acts as a tool to structure a musical piece (Calvert and Tart, 1993; Moussard, Bigand, Belleville, and Peretz, 2012). Moussard, Bigand, Belleville, and Peretz (2012) explain that "the 
rhythm...can give cues for the lyric's structure (such as the number of syllables per line) and limit the possible choices among the words that can be set to the melody" (p. 521). The rhythm of a piece works to guide the lyrics, potentially working as a subtle, constant reminder of structure, helping to create a cohesive musical and aesthetic experience.

\subsection{Music, Memory, and Information Retention}

This section of the literature review explores scholarly works that consider the relationship between music, memory, and information retention. Understanding the relationship between these elements is crucial, since the creation of the three musical compositions for this project are primarily based upon this area of research. The following articles discuss music's potential to be a rehabilitation tool that assists with memory and information retention, primarily using studies that consider the relationship between music and patients with Alzheimer's disease, dementia, or other variations of memory loss. Although much of the research included in this section relates to Alzheimer's patients, awareness of the influence music has on individuals with Alzheimer's disease assists in informing the creation of compositions that aim to aid individuals with varied forms of memory loss in recalling information.

In Young's (2013) article, she discusses the relationship between music and people with Alzheimer's and other dementias or variations of memory loss. She explores the idea of re-creative music therapy, which is multifaceted. One aspect of re-creative music therapy is 'music 
reminiscence groups,' in which 'the music therapist uses live and/or recorded precomposed music to enhance and/or promote participation in reminiscence discussion groups" (Young, 2013, p. 730). Another aspect is 'lyric analysis,' in which "the music therapist uses lyrics from familiar songs (verbally and musically) to convey a positive message or to validate a client's feelings" (Young, 2013, p. 730). Although there is currently no cure for most dementias, Young (2013) demonstrates the importance of music as a potentially communicative and rehabilitating tool that could be used to assist patients with varied forms of memory loss.

In line with Young (2013), Moussard et al. (2012) show interest in music's influence on memory and information retention. Their study questions whether music assists in the learning and memorization of new lyrics, using a 68-year-old patient with mild Alzheimer's disease as a basis for their study (Moussard et al., 2012). The authors test the memorization ability of the participant in the following conditions: "(1) spoken, (2) sung on a non-familiar (NF) melody, (3) sung on a recently learned (low familiar, LF) melody, and (4) sung on a life-long and highly familiar (HF) melody" (Moussard et al., 2012, p.523). The results of the study indicate that in the early stages of learning lyrics, singing does not assist with memorization of the lyrics and is more difficult to learn when the melody was unfamiliar (Moussard et al., 2012). But after several learning sessions, the results demonstrate an increase in memorization ability (Moussard et al., 2012). Moussard et al. (2012) additionally note that when lyrical recall is "delayed 
by one month (week 9) or even 10 [minutes], sung words are better recalled than spoken words" (p. 528), supporting the power of music as a factor that assists in verbal retention and validating the use of music as a communicative tool.

Other research in this field offers similar results, supporting the use of music as a factor that assists in information retention and memory recall (El Haj, Antoine, Nandrino, Gély-Nargeot, and Raffard, 2015; Palisson et al., 2015; Simmons-Stern, Budson, and Ally, 2010). Simmons-Stern, Budson, and Ally (2010) explain that "the daughter of an [Alzheimer's disease] patient ... described successfully teaching her father current events by singing news stories to the tune of popular songs" (p. 3164). Simmons-Stern, Budson, and Ally (2010) examine whether music has an influence on memory in patients with Alzheimer's and if it can assist the learning process. Using children's songs (unfamiliar to the patients) with repetitive melodies as a basis for their analysis, the authors state that patients with Alzheimer's could recall lyrics of songs better when they were accompanied by a sung recording rather than a spoken recording, implying that music can be an effective tool to teach information to those with memory-loss diseases (Simmons-Stern, Budson, and Ally, 2010). Furthermore, they note that future studies in this area can investigate "the possibility of presenting practical, every-day information to patients with [Alzheimer's disease] in the form of song" (Simmons-Stern, Budson, and 
Ally, 2010, p. 3166), which is what this project's creative compositions aim to do.

According to Calvert and Tart (1993), the nature of songs and the repetition present within them causes the melody of a song, along with the Iyrical content, to be easily rehearsed and remembered. Calvert and Tart (1993) emphasize the communicative power of music, noting that elements within a composition, such as a constant rhythm denoting a pattern, assist with this retention. The inclusion of a steady rhythmic pattern was an important consideration to be aware of during the creation of this project's musical compositions.

Taking a slightly different approach, Eschrich, Münte, and Altenmüller (2008) analyze the relationship between emotional music and memory. Their study examines the influence of music on memory, exploring whether emotional music is better remembered than music that is less emotional (Eschrich, Münte, and Altenmüller, 2008). Eschrich, Münte, and Altenmüller (2008) state musical pieces that are heavily emotional (those that induced high levels of arousal) are better remembered by listeners than those that are less emotional. These results suggest that emotion present in a musical piece is critical to the retention of ideas presented in that musical piece.

Samson, Dellacherie, and Platel (2009) also show interest in the influence emotion present in musical compositions has on memory. More specifically, their four studies determine the extent that emotion in music 
can impact memory. The first is concerned with a comparison between emotion, music, and memory in patients with temporal lobe epilepsy (TLE) and those without. In the first study on participants without TLE, the authors conclude that emotion present in a song - specifically the emotions of fear and happiness, which have high arousal levels - can strengthen the recollection of the song presented with said emotion (Samson, Dellacherie, and Platel, 2009). These results differ in the second study which examine eight patients with temporal lobe epilepsy who were non-musical, in this case noting that peaceful music was better remembered over "contexts with high arousal, such as happiness and to some extent fear, [which] do not appear to enhance memory as compared to excerpts with low arousal level" (Samson, Dellacherie, and Platel, 2009, p. 249). The authors hypothesize that this reaction to peaceful, low arousal level music could be a result of lowering the listener's level of anxiety, assisting in enhancing their memory (Samson, Dellacherie, and Platel, 2009). Following the first two studies are two more that specifically relate to musical memory in patients with moderate to severe Alzheimer's disease. The first of the two studies examine familiarity of strictly verbal compositions, strictly musical compositions, and compositions that paired music with lyrics, experienced through repeated exposure over an extended period of time (Samson, Dellacherie, and Platel, 2009). The results of the study suggest that excerpts that include "musical stimuli [produce] a stronger feeling of familiarity than verbal ones" (Samson, Dellacherie, and Platel, 2009, p.251). 
The second of the two studies focuses on long-term retention of poems and musical compositions, along with the ideas and emotions presented in those compositions (Samson, Dellacherie, and Platel, 2009). The findings indicate that musical compositions are better remembered than verbal poems (Samson, Dellacherie, and Platel, 2009). In addition, the authors note that participants can distinguish between the happy and sad emotions present in the compositions, with happy compositions moving and arousing listeners more than sad compositions (Samson, Dellacherie, and Platel, 2009). All of these results indicate that emotion influences memory of musical compositions; further suggesting that emotion present in a musical piece could assist a listener's memory of not only the musical piece itself, but also the information presented in that musical piece.

\section{Methodology}

The primary method being used in this research-creation project is the research-creation methodology. A research-creation project is one that "typically [integrates] a creative process, experimental aesthetic component, or an artistic work as an integral part of a study" (Chapman and Sawchuk, 2012, pg. 6). The research-creation methodology has been recognized relatively recently, becoming more widely used in the areas of digital humanities and social sciences within the past 15 years (Chapman and Sawchuk, 2012). Chapman and Sawchuk (2012) describe four different 
types of research-creation, including: "'research-for-creation," "researchfrom-creation," "creative presentations of research," and finally, "creationas-research"'” (pg. 7). For this research-creation project, I used the method of "creative presentations of research," (Chapman and Sawchuk, 2012) as this project's compositions are a result of scholarly research gathered during the study. A large amount of literature has informed the creation of the three compositions, but only those that refer to specific musical elements and the impact they have on communicating emotion in a composition, and whether they can influence retention were used to guide the creation of the compositions.

The literature gathered for this project was primarily found online using ScienceDirect, EBSCOhost, and Google Scholar, searching key terms such as "Music and emotion," "Music and advertising," "Music and cognition," "Music and Alzheimer's disease," "Music and (retention of information)," and "Information retention and emotion". Initial searches in these databases yielded a wide range of results. As a result, those that did not speak specifically to the communicative influence of specific musical traits were excluded. Informed by sampling techniques used in empirical research methods, articles were sampled on a non-probability basis, choosing articles based on the factor of being purposive (those that were relevant to the research-creation) ("How To... Conduct Empirical Research," n.d.). 
In order to inform the research-creation methodology, an inductive literature analysis was performed, examining existing text-based research in the field. Determining the extent to which research has developed in this field through this analysis has strengthened my research and offered insights that contributed to this study and the creation of its creative components. The findings associated with the observations have contributed to an understanding of this area of research, informing my viewpoint related to the appropriate methods and musical techniques to use when communicating with individuals through music. It has also contributed to an understanding of research areas that could be explored in the future.

\section{The Compositions: An Overview}

This research-creation project considers the ways that music can be used to assist in aiding verbal memory and communicating emotion, and includes three original musical compositions that were developed in response to the scholarly literature on this topic. The three musical compositions that have been produced for this project, each between 50 and 100 seconds in length, aim to communicate emotion, create aesthetic satisfaction, and aid in reinforcing the production of memories based on the information presented in the compositions.

There are five elements that these compositions have in common: (1) they are all composed in a 4/4 time signature, (2) they may share chords 
but no two compositions will have identical chord progressions, (3) they each use a different primary instrument, (4) they each aim to elicit one of the three emotions discussed by Fritz et al. (2009); happy, sad, or scared/fearful, and (5) they are all between 50 and 100 seconds long. The primary instruments used in the compositions are: (1) ukulele (2) guitar, and (3) piano. These were the primary instruments chosen for the compositions as they each have a different timbre (tone), and are three instruments that I have experience playing and composing with. Although each musical composition uses a particular primary instrument, additional background instruments such as drums and violin were used in each composition. The background instruments included in the compositions are synthesized sounds made to sound like original instruments, and were found in the Apple GarageBand library. Although these background instruments are synthesized representations of instruments, they are original arrangements that were composed using a keyboard.

\subsection{Composition Influences from Literature}

This project's creative compositions were informed by content gathered from existing literature in the field discussing musical elements and the impact they have on the way a musical piece is heard and perceived. The following section is a discussion of scholarly work from authors that speak to the communicative power of music, and the influence that particular musical elements can have on aiding memory and assisting with information retention. As mentioned above, the literature gathered for 
this project was primarily found on Google Scholar, ScienceDirect, and EBSCO, using a variety of different key terms related to this area of research. As initial searches in these databases yielded a wide range of results, those that did not specifically speak to the communicative influence of specific musical elements were excluded. Informed by sampling techniques used in empirical research methods, articles were sampled on a non-probability basis, choosing articles based on the factor of being purposive (those that were relevant to the research-creation) ("How To... Conduct Empirical Research," n.d.).

\subsubsection{Emotion.}

Eschrich, Münte, and Altenmüller (2008) found musical pieces that were heavily emotional were better remembered than those that were less emotional. The stimulation associated with heavily emotional musical pieces (Eschrich, Münte, and Altenmüller, 2008; Samson, Dellacherie, and Platel, 2009) indicates the importance of emotion present in the musical compositions created for this study.

Furthermore, Juslin and Laukka's (2001) study on the impact vocal intonation has on perceived emotions in a listener was important to consider when creating these compositions. Their study suggested that vocal expression influences the perceived emotion of what is being stated (Juslin and Laukka, 2001). Specific acoustic cues (i.e. high-frequency energy) foreshadowed the emotion intensity rating results in their study (Juslin and Laukka, 2001). This has informed the compositions, as there is clear 
evidence suggesting the importance of vocal expression when attempting to communicate a specific emotion.

\subsubsection{Chords used and tempo.}

The chosen musical mode of the compositions was based on discussion in Vieillard et al.'s (2008) article, which addressed the pairing between musical structure in a composition and the emotion a composition aims to elicit. For the sake of their study, they classified happy compositions as having a fast tempo in a major mode, sad compositions as having a slow tempo in a minor mode, and threatening compositions as having an intermediate tempo in a minor mode (Vieillard et al., 2008). For the sake of my compositions, scared/fearful has been made interchangeable with Vieillard et al.'s (2008) classification of threatening, therefore the scared/fearful composition has an intermediate tempo in a minor mode. Simmons-Stern, Budson, and Ally (2010) noted that future studies in the area of music and memory loss could investigate "the possibility of presenting practical, every-day information to patients with [Alzheimer's disease] in the form of song," (Simmons-Stern, Budson, and Ally, 2010, p. 3166) which is what these compositions aim to do.

\subsubsection{Primary instrument.}

Poulin-Charronnat (2004) emphasized the impact the instrumentation in a musical piece could have on the way it is perceived. The focus of Poulin-Charronnat's (2004) experiment was to uncover 
whether or not the timbre associated with a musical instrument impacts the way a listener recognizes and remembers a musical piece. The results of the study suggested that a change in the timbre (tone) of a musical instrument in a piece has substantial impact on the way the excerpt was remembered (Poulin-Charronnat, 2004). Poulin-Charronnat (2004) explained that "the detrimental effect of timbre change on recognition for musicians demonstrates that timbre qualities are indeed integrated into this trace, because performance deteriorates drastically when the instrumentation changes between the memorized excerpt and the to-berecognized excerpt, even though listeners were instructed to ignore such changes" (p. 248). By this, Poulin-Charronnat (2004) was explaining that the memory of a musical composition was closely tied to the timbre and tone of the instruments used in the composition. If the timbre and tone of a composition were to change (i.e. if a piano was used to play a piece originally played on a guitar), this would hinder an individual's ability to adequately remember a composition and the ideas present within it.

This could have implications for future research in this area beyond the scope of this MRP. When testing the effectiveness of these compositions, it will be important to use the same instrument continually (or play an exact recording of the composition) when examining the piece's communicative ability. In general, this could be a useful point to be aware of for those playing musical compositions to those who have experienced varied forms of memory loss. If an individual were to play and sing musical 
excerpts on a guitar in an attempt to evoke memory of the verbal message within the musical piece to an individual who suffers from a type of memory loss, the evidence put forth by Poulin-Charronnat (2004) suggests that it would be important to use the same instrument each time the composition is played.

For the sake of the compositions created for this project, the primary instruments of ukulele, guitar, and piano were chosen based on their different timbre (tone), and the fact that they are three instruments I have experience playing and composing with.

\subsubsection{Message.}

The message of these compositions was not informed by any particular scholarly work, but was informed by personal knowledge of the issues faced by my family member who has dementia.

\subsubsection{Lyrics.}

The lyrics in each composition were created with repetition (Hargreaves, 1984; Ockelford 2006; 2009; 2013) and rhyming in mind, as these traits emphasize music's ability to communicate and become ingrained in the memory of the listener. A rhyme, specifically an end rhyme, in a technique that is often used in poetry (Hyman and Rubin, 1990), and one found in popular music. Repetition of exposure to repeated melodies has been demonstrated to assist with memory in patients with memory loss 
(Moussard, Bigand, Belleville, and Peretz, 2012). This could potentially assist in retention of the information presented in the compositions.

In addition, Moussard, Bigand, Belleville, and Peretz (2012) discussed the notion of rhythm giving cues for lyrical structure, directing the listener to be able to logically follow along with the musical composition. For this reason, the melody of the lyrics and the melody of the lead instrument in the composition share a similar rhythmic pattern. This could additionally relate to classical conditioning theory (Bierley, McSweeney, and Vannieuwkerk, 1985). For example, if a listener hears the melody of the piano in Composition $\mathrm{C}$ without the lyrics (after repeated exposure), or finds themselves humming the tune of the composition, they may still be impacted by the message on a subconscious level.

As mentioned in my introduction, inspiration for the lyrics of the three compositions was inspired by two factors: a personal family member's difficulty with everyday tasks (e.g., brushing his teeth, shaving, etc.) and a detail mentioned in Simmons-Stern, Budson, and Ally's (2010) article describing the daughter of an Alzheimer's patient using music to teach her father about current news stories.

\subsection{Composition Breakdown}

Below is a specific breakdown of each composition, consisting of the emotion each piece aims to portray, the chords used in the composition, the tempo, the primary instrument, the message of the composition, the lyrics in the composition, and discussion of the chosen lyrics. Different colours 
are used to signify where rhyming and repetition is present in each piece. Each composition breakdown has a section titled 'Lyrics in the composition,' which is where these different colours will be visible. Green represents an end rhyme; for a rhyme to be green, it must rhyme with the end rhyme in the line above, or the end rhyme in the line below. Orange represents a prominent word used as an adjective that is repeated throughout the composition; for a word to be orange, it must be mentioned at least three times. Blue represents the repetition of an entire line; for a line to be blue it must be an exact lyrical replication of an existing line. There is also repetition present within the chords used in each composition. At the end of each lyrical line, there will be a list of the musical chords used in that line. Red represents repetition of chords used from line to line; for the chords to be red, they must be an exact replication of the line above or below it. The chords listed are the base chords that the lead melody was created over.

\title{
Composition A:
}

\author{
Emotion: Happiness (Fritz et al., 2009). \\ Chords Major mode (Vieillard et al., 2008); D major, A major, E minor, G \\ used: \\ major. \\ Tempo: $\quad$ Fast tempo (Vieillard et al., 2008). \\ Primary Ukulele \\ instrument: \\ Message: Communicating about brushing one's teeth. \\ Lyrics in the When I wake up in the morning, I stand up (D, A, Em, G) \\ composition:
}


I walk down the hall to the bathroom, and grab my cup (D, A, Em, G)

I have a sip of water, then pick up toothbrush $(A, G)$

I have a sip of water, then pick up toothbrush $(A, G)$

My toothbrush is yellow, my toothpaste is blue (D, A, Em, G)

My mouth is now open and my hand begins to move (D, A, Em,

G)

My toothbrush is yellow, my toothpaste is blue (D, A, Em, G)

And remember to rinse and spit too $(A, G)$

Put your tooth brush back and now you leave the room (A, G)

That's what you do (A, D)

Lyrical

The lyrics in this composition aim to communicate the routine of

discussion:

brushing one's teeth. The lyrics are not complex, using simple words and an easy-to-follow melody. Within the lyrics, the colour of the toothbrush or toothpaste, for example, could be changed to fit an individual's preference or situation. As repetition and rhyming were evidenced to assist with memory (Hargreaves, 1984; Hyman and Rubin, 1990, Calvert and Tart, 1993) there is a lot of repetition and rhyming in this composition in the lyrics, the melody, and the chords used in the composition.

\section{Composition B:}

Emotion: $\quad$ Sadness (Fritz et al., 2009). 
Chords

used:

Tempo:

Primary instrument:

Message:

Lyrics in the composition:
Minor mode (Vieillard et al., 2008); A minor, E minor, G major, D major.

Slow tempo (Vieillard et al., 2008).

Guitar

Communicating about loss, specifically the loss of a loved one.

Where did the time go? I can't recall (Am, Em, G, D)

Seems like yesterday we were arm in arm, walking through the halls (Am, Em, G, D)

Where did the time go? I can't recall (Am, Em, G, D)

You were here before, and we had it all (Am, Em, G, D)

Goodbye baby, goodbye love (Am, Em, G, D)

All our memories, forever in my heart (Am, Em, G, D)

Goodbye baby, goodbye love (Am, Em, G, D)

Though the pain is constant, l'll rise above (Am, Em, G, D)

Goodbye love (Am, Em, G, D)

Lyrical discussion:
The lyrics in this composition aim to communicate about the loss of a loved one. As opposed to the lyrics in Composition A, which were more instructional, the lyrics in this composition are using the emotion of sadness to set the tone for contemplation of life, love, and loss. Similar to Composition A, the lyrics in this composition are not complex, using simple words and an easyto-follow melody. Repetition and rhyming were also heavily 
prominent in the lyrics, the melody, and the chords used in this composition.

\section{Composition C:}

Emotion: $\quad$ Scared/fearful (Fritz et al., 2009).

Chords Minor mode (Vieillard et al., 2008); A minor, D minor, E minor, G used: major.

Tempo: Intermediate tempo (Vieillard et al., 2008).

Primary Piano

instrument:

Message: Communicating about hurting oneself, specifically the risk associated with touching hot surfaces.

Lyrics in the The oven's hot, yeah lots of things are hot (Am, Dm) composition:

That's something we should know (Am, Dm)

The oven's hot, yeah lots of things are hot (Am, Dm)

Don't want to touch the stove (Am, Dm)

If you want to make some tea (Em, Dm)

If you want to iron your shirt sleeve (Em, Dm)

Just ask for help because these things can be (Em, Dm)

Hot! (Em, Am)

These things are hot $(\mathrm{Am}, \mathrm{Em})$

These things are hot (Am, Em)

These things are hot (Am, Em)

Some things are hot (Am, Em) 
Lyrical The lyrics in this composition aim to communicate the risks discussion: associated with contacting hot surfaces. Similar to Composition

A, the lyrics of this composition were purposefully instructional, with the goal of having the listener be aware of the dangers associated with touching hot surfaces. The word "hot" was used multiple times throughout the composition to emphasize the message of the song, with repetition of the phrase "things are hot" at the end of the composition to further accentuate the message.

\section{Discussion}

The following section includes a discussion related to the creative process that occurred when producing the compositions, along with an individual composition summary.

\subsection{General Notes on Creating the Compositions}

As mentioned, according to Calvert and Tart (1993), the nature of songs and the repetition present within them causes the melody of a song, along with the lyrical content, to be easily rehearsed and remembered. Consequently, repetition was an important factor that needed to be included to not only support memory recall, but also to create aesthetic satisfaction. When there is repetition present in a musical composition, there is a 
constant sense of expectation being met, leading to a feeling of aesthetic satisfaction and resolution (Ockelford 2006; 2009; 2013).

When creating the compositions, it was difficult to ensure the lyrics in each composition effectively communicated the desired message to the intended adult audience in a way that did not come across as puerile. The challenge to find a balance between creating compositions for a mature, adult audience, while also recognizing these compositions were being created for individuals with dementia or Alzheimer's disease, was primarily concerning when creating Compositions $\mathrm{A}$ and $\mathrm{C}$, as they were very instructional. Though the goal was to ensure the melody and lyrics in each composition were uncomplicated (to assist with memory recall), future research should consider what level of lyrical complexity is appropriate for different spectrums of memory loss. This would ensure the message within the composition could be appropriately communicated to each group on the spectrum in the most effective way.

Although the instructional nature of Composition A and Composition $\mathrm{C}$ resulted in the aforementioned communication concern, of the three compositions, Composition $\mathrm{A}$ and $\mathrm{C}$ were the easiest to compose. They both came together very quickly, and the lyrics effectively depict the message that the composition aims to portray. Composition B was much more difficult to create, as the message of the song was less specific than the other two compositions, and as a result, was more difficult to capture through the lyrics. There was no specific desired action associated with 
Composition $\mathrm{B}$, whereas Composition $\mathrm{A}$ and $\mathrm{C}$ were asking the listener to remember how to brush their teeth, or to be aware of the risks associated with touching hot surfaces. The lack of a call to action in the lyrics, and the more abstract nature of the message that Composition B was attempting to portray made the lyrics more difficult to write.

In regards to the emotion each composition was aiming to portray, the emotion of scared/fearful was the most difficult to depict. The musical chords used for the emotion of happy (primarily major chords in a major key) and the emotion of sad (primarily minor chords in a minor key) lent themselves more naturally to the emotion they were trying to portray. However, the emotion of scared/fearful (Composition C) relied on much more than just the key it was created in to adequately depict the emotion. For instance, other musical factors, like the dynamics (how quiet or loud an instrument is being played) in the piece played an important role in illustrating the emotion of Composition C. Although factors such as dynamics played an important role in the portrayal of the emotions in Compositions $\mathrm{A}$ and $\mathrm{B}$, Composition $\mathrm{C}$ relied more on a change in dynamics to emphasize the chaos associated with feeling scared/fearful, whereas $A$ and $\mathrm{B}$ did not. For example, in Composition B a decision was made to play the guitar using a softer, plucking pattern, rather than a heavy, strumming pattern. The chosen dynamic of Composition B was consistent throughout the piece, along with the chosen dynamic of Composition A. Contrary to A and $\mathrm{B}$, Composition $\mathrm{C}$ has a change in dynamics throughout the piece, 
resulting in a more chaotic sound. The manipulation of the dynamics in Composition $\mathrm{C}$ worked to create a sense of disorder, further emphasizing the feeling of being scared/fearful.

\subsection{Individual Composition Summary}

Composition A. Creating this composition in a major mode with primarily major chords, as informed by Vieillard et al. (2008), resulted in the composition sounding happy, which is the emotion this composition aimed to elicit. The timbre of the ukulele in this composition is very bright, as it is a higher-pitched instrument, again reinforcing the notion that this is a happysounding composition. In addition, a faster tempo was used, taking cues from Vieillard et al.'s (2008) study, which assisted in the composition conveying the desired emotion. As mentioned by Eschrich, Münte, and Altenmüller (2008), musical pieces that were heavily emotional (those that induced high levels of arousal) were better remembered by listeners than those that were less emotional (inducing lower levels of arousal). This composition was created with that in mind, pairing a faster tempo with an upbeat melody in hopes that this resulted in a production of high levels of arousal in the listener.

The background instruments supported the theme and emotion this composition aimed to communicate. The bass guitar contributed to the steady rhythm of the composition, changing notes every other beat in a continuous pattern. A change in the drum beat occurred as the composition entered the second section of the song ("My toothbrush is yellow, my 
toothpaste is blue,") which will be referred to as the 'chorus'. In the chorus of this composition, the lyrics and the melody of the vocals are the most repetitive, which is why the most important aspect of the message, remembering to use the yellow toothbrush and blue toothpaste, is included in this section. In addition, the vocal melody changes in the chorus. As so, I chose to change the drum beat to emphasize the differentiation between these two parts.

Composition B. Creating this composition in a minor mode, with primarily minor chords, assisted in the composition sounding sad. The warm timbre of the guitar paired with the softer playing of the instrument (plucking as opposed to heavy strumming) emphasized the melancholic features typically associated with sadness. The slow tempo, inspired by Vieillard et al.'s (2008) classification of sad compositions as having a slow tempo, also worked to portray the desired emotion. Taking cue from Juslin and Laukka's (2001) study, which suggested that vocal expression influences the perceived emotion of what is being stated, the vocals in this composition were purposefully sung very calmly, incorporating raspy vocal qualities throughout the piece to emphasize the emotion this composition aimed to portray.

In addition, the background instruments supported the theme and emotion this composition aimed to communicate. The inclusion of synthetic strings (violin, cello, double bass) in the background of this composition worked to emphasize the melodic and melancholic features within the song, 
further emphasizing the emotion of sadness that this composition aimed to communicate. The rich timbre of the strings paired well with the warm lead guitar and vocals.

Similar to Composition A, there was a change in the drum beat as the song entered the chorus ("Goodbye baby, goodbye love"). The differentiation between the two sections of this song was also marked by a repetitive guitar note that elevated the entrance into the chorus. As the lyrical message in the chorus of the composition summarizes the message the entire piece aims to communicate, it was important to emphasize this section.

Composition C. Pairing this composition with the chosen chord progression of all minor chords assisted in accentuating the scared/fearful emotion the composition aimed to portray. In addition, the more aggressive tone, volume, and overall vocal expression of the lead vocal, informed by Juslin and Laukka (2001), worked to create a feeling of tension in the listener, supporting the scared/fearful emotion this piece aimed to elicit from the audience. In this piece, there are moments where the vocals are purposefully louder, specifically in the last few lyrical phrases when the word "hot" is sung. It was important to emphasize "hot" throughout the composition, using factors such as repetition and increased volume to do so, as the message this composition aimed to communicate was about the risks associated with touching hot surfaces. 
The background instruments, also worked to accentuate the word "hot." A cymbal was also added every time "hot" was sung, which emphasized the message while also creating a change in dynamics within the piece, adding to the chaotic sound. The manipulation of the dynamics in Composition $\mathrm{C}$ worked to create a sense of disorder, further emphasizing the feeling of being scared/fearful.

In this composition, the rhythm of the piano gave cues for the lyrical structure (Moussard, Bigand, Belleville, and Peretz, 2012), guiding the lyrics and working as a constant reminder of the vocal melody, helping to create a cohesive musical and aesthetic experience. As a result of the melody closely following the lead vocal the hope is that, after repeated exposure to the composition, an individual could listen to the lead piano without the lyrics and recall the entire message of the song.

The intermediate tempo of the composition, inspired by Vieillard et al.'s (2008) classification, was an aspect that was challenging to incorporate. When creating this particular composition, I experienced a desire to use a faster tempo in an attempt to add to the chaotic feeling associated with being scared/fearful. This leads me to believe that an intermediate tempo for this composition may not have been the most effective. This lack of a cohesive pairing between the tempo and chosen emotion could be a result of Vieillard et al.'s (2008) classification referencing the emotion of threatening, rather than the emotion of scared/fearful. For the sake of this MRP, the emotions of threatening and scared/fearful were 
interchangeable. This is an aspect that would be reconsidered in the future if additional compositions were to be created with the same objective.

\section{Future Implications and Research}

Although it is beyond the scope of this project, these compositions were created with the intention that they be deployed for the purposes of enhancing the memory of listeners in the future. As a result, thought was given to the ways that the compositions could be tested and used as a method to understand the ways that music can communicate to individuals with varied forms of memory loss, assisting in information retention. To allow for more complex testing and analysis of the compositions, each composition was created with the inclusion of specific traits (emotion, particular instruments, etc.) to serve as a basis for evaluating the communicative power of the compositions. Upon hearing the compositions repeatedly, the participants of the study would be asked to rate the various aspects of the compositions based on their ability to: (1) effectively communicate emotion, (2) effectively communicate the idea presented in the lyrics, (3) be aesthetically pleasing, and (4) assist with cognitive comprehension and information retention. 


\section{Conclusion}

This MRP/research-creation project explored music as a communicative medium, specifically the way that music can assist in cognitive comprehension and information retention in individuals with memory loss. Scholarly literature that discusses the influence of music, the presence of emotion in music, how musical elements can be used to facilitate communication, and the relationship between music and memory was used to inform the creation of three musical compositions designed to communicate emotion, create aesthetic satisfaction, and aid in recollection and memory of the information presented in the compositions. Using a research-creation method for this project has allowed for the production of material that could be utilized to develop further studies in this area of research in the future. 


\section{References}

Alpert, J. I., \& Alpert, M. I. (1990). Music influences on mood and purchase intentions. Psychology \& Marketing, 7(2), 109-133.

Apaolaza-Ibáñez, V., Zander, M., \& Hartmann, P. (2010). Memory, emotions and rock ' $n$ ' roll: The influence of music in advertising, on brand and endorser perception. African Journal of Business Management, 4(17), 3805-3816.

Bierley, C., McSweeney, F. K., \& Vannieuwkerk, R. (1985). Classical conditioning of preferences for stimuli. Journal of Consumer Research (Pre-1986), 12(3), 316- 323.

Calvert, S. L., \& Tart, M. (1993). Song versus verbal forms for very-longterm, long-term, and short-term verbatim recall. Journal of Applied Developmental Psychology, 14(2), 245-260. doi:10.1016/01933973(93)90035-T

Chapman, O., \& Sawchuk, K. (2012). Research-Creation: Intervention, analysis and "family resemblances". Canadian Journal of Communication, 37(1), 5-26.

El Haj, M., Antoine, P., Nandrino, J. L., Gély-Nargeot, M., \& Raffard, S. (2015). Self- defining memories during exposure to music in alzheimer's disease. International Psychogeriatrics / IPA, 27(10), 1719-12. doi:10.1017/S1041610215000812

Eschrich, S., Münte, T. F., \& Altenmüller, E. O. (2008). Unforgettable film music: The role of emotion in episodic long-term memory for music. BMC Neuroscience, 9(1), 9-48. doi:10.1186/1471-2202-9-48

Fritz, T., Jentschke, S., Gosselin, N., Sammler, D., Peretz, I., Turner, R., ... \& Koelsch, S. (2009). Universal recognition of three basic emotions in music. Current biology, 19(7), 573-576.

Gorn, G. J. (1982). The effects of music in advertising on choice behavior: A classical conditioning approach. Journal of Marketing, 46(1), 94101.

Hargreaves, D. J. (1984). The effects of repetition on liking for music. Journal of research in Music Education, 32(1), 35-47.

"How To... Conduct Empirical Research." How To...Conduct Empirical Research Part: N.p., n.d. Web. 2 June 2017. 
Hyman, I. E. J., \& Rubin, D. C. (1990). Memorabeatlia: A naturalistic study of long-term memory. Memory \& Cognition, 18(2), 205.

Ilie, G., \& Thompson, W. (2006). A Comparison of Acoustic Cues in Music and Speech for Three Dimensions of Affect. Music Perception: An Interdisciplinary Journal, 23(4), 319-330.

doi:10.1525/mp.2006.23.4.319

Juslin, P. N., \& Laukka, P. (2001). Impact of intended emotion intensity on cue utilization and decoding accuracy in vocal expression of emotion. Emotion, 1(4), 381-412. doi:10.1037//1528-3542.1.4.381

Kellaris, J. J., \& Cox, A. D. (1989). The effects of background music in advertising: A reassessment. Journal of consumer research, 16(1), 113- 118.

Koelsch, S. (2010). Towards a neural basis of music-evoked emotions. Trends in Cognitive Sciences, 14(3), 131-137. doi:10.1016/j.tics.2010.01.002

Livingstone, S. R., \& Thompson, W. F. (2006). Multimodal affective interaction: A comment on musical origins. Music Perception, 24(1), 89-94. doi:10.1525/mp.2006.24.1.89

Morris, J. D., \& Boone, M. A. (1998). The effects of music on emotional response, brand attitude, and purchase intent in an emotional advertising condition. NA-Advances in Consumer Research, 25(1), 518-526.

Moussard, A., Bigand, E., Belleville, S., \& Peretz, I. (2012). Music as an aid to learn new verbal information in alzheimer's disease. Music Perception: An Interdisciplinary Journal, 29(5), 521-531. doi:10.1525/mp.2012.29.5.521

Ockelford, A. (2006). Implication and expectation in music: A zygonic model. Psychology of Music, 34(1), 81-142. doi:10.1177/0305735606059106

Ockelford, A. (2009). Similarity relations between groups of notes: Musictheoretical and music-psychological perspectives. Musicae Scientiae, 13(1Suppl), 47-98. doi:10.1177/102986490901300104

Ockelford, A. (2013). Applied musicology: Using zygonic theory to inform music education, therapy and psychology research. New York: Oxford University Press.

Palisson, J., Roussel-Baclet, C., Maillet, D., Belin, C., Ankri, J., \& Narme, P. (2015). Music enhances verbal episodic memory in alzheimer's 
disease. Journal of Clinical and Experimental

Neuropsychology, 37(5), 503.

doi:10.1080/13803395.2015.1026802

Panksepp, J., \& Bernatzky, G. (2002). Emotional sounds and the brain:

The neuro-affective foundations of musical appreciation.

Behavioural Processes, 60(2), 133-155. doi:10.1016/S03766357(02)00080-3

Patel, A. D. (2006). Musical rhythm, linguistic rhythm, and human evolution. Music Perception: An Interdisciplinary Journal, 24(1), 99104.

Patel, A. D., \& Daniele, J.R. (2003). An empirical comparison of rhythm in language and music. Cognition, 87(1), 35-45.

Repp, B. H. (2005). Sensorimotor synchronization: a review of the tapping literature. Psychonomic bulletin \& review, 12(6), 969-992.

Révész, G. (1954). Introduction to the psychology of music. Courier Corporation.

Poulin-Charronnat, B. (2004). Effects of a change in instrumentation on the recognition of musical materials. Music Perception: An Interdisciplinary Journal, 22(2), 239-263.

doi:10.1525/mp.2004.22.2.239

Samson, S., Dellacherie, D., \& Platel, H. (2009). Emotional power of music in patients with memory disorders: Clinical implications of cognitive neuroscience. Annals of the New York Academy of Sciences, 1169(1), 245-255. doi:10.1111/j.1749-6632.2009.04555.x

Scherer, K. R. (2004). Which emotions can be induced by music? What are the underlying mechanisms? And how can we measure them? Journal of new music research, 33(3), 239-251.

Scott, L. (1990). Understanding Jingles and Needledrop: A Rhetorical Approach to Music in Advertising. Journal of Consumer Research, 17(2), 223-236.

Simmons-Stern, N. R., Budson, A. E., \& Ally, B. A. (2010). Music as a memory enhancer in patients with alzheimer's disease.

Neuropsychologia, 48(10), 3164-3167. doi:10.1016/j.neuropsychologia.2010.04.033

Vieillard, S., Peretz, I., Gosselin, N., Khalfa, S., Gagnon, L., \& Bouchard, B. (2008). Happy, sad, scary and peaceful musical excerpts for 
research on emotions. Cognition \& Emotion, 22(4), 720-752. doi:10.1080/02699930701503567

Wellek, A., \& Baker, T. (1926). Quarter-Tones and Progress. The Musical Quarterly, 12(2), 231-237.

Young, L. (2013). Persons with Alzheimer's Disease and Other Dementias. In E. Lillian, Guidelines for Music Therapy Practice in Mental Health (pp. 718-766). Gilsum, NH: Barcelona Publishers. 